\title{
Radiation Detection with Distributed Sensor Networks
}

\author{
Sean M. Brennan, Arthur B. Maccabe, Angela M. Mielke, David C. Torney
}

\begin{abstract}
The illegitimate transport of radioactive material is a national security issue which is typically addressed by portal radiation monitors positioned at chokepoints. Such a solution is not always practical however, so we are investigating the application of multiple, smaller radiation detectors incorporated in a distributed sensor network. Preliminary research and simulation indicates that this approach offers greater discretion and easier deployment with little or no loss of accuracy.
\end{abstract}

Index Terms- discrete event simulation, coherent addition, nuclear nonproliferation, Bayesian detection

\section{INTRODUCTION}

W HEN considering the risks to our vulnerable populations posed by potential terrorism, the nuclear threat is never far from center stage. Although weapons-grade nuclear materials are heavily guarded, a rather plausible scenario involves the detonation of a very simple Radiological Dispersion Device (RDD) which is capable of broadcasting non-fissile but highly radioactive particles over a densely populated area. In most cases, such a device and its payload must be transported to a target destination. Detection and intervention is the final defense in such a situation. Currently large portal monitor systems placed at traffic choke-points facilitate the detection of illicit isotopes. A number of these are already in use at various sites in the United States.

The Distributed Sensor Network (DSN) project at Los Alamos National Laboratory, in cooperation with the University of New Mexico, is investigating the use of a network of multiple radiological detectors coupled with other sensors with supportive data collection for RDD detection. Such a scattered system is far more suitable to the urban environment that is the prime target in this scenario. A DSN for dispersed radiological detection complements the portal monitor solution by being deployable in areas

Manuscript submitted on February 2, 2004. where the latter is not practical. The DSN option is much less visible, uses less power per detector, is more rapidly deployed (since each unit can be handcarried), and simplifies coverage over the variety and multitude of transport avenues into an urban center. Also, portal monitoring systems typically require slow or halted traffic to function effectively, whereas our DSN approach can be tailored for any moderate traffic speed with no loss of effectiveness.

Our goal is to demonstrate the performance of our DSN approach to radiation detection, particularly in comparison to the performance of typical portal monitors. We will soon accomplish this by a physical demonstration of a small DSN system and by large scale demonstrations via validated simulation. The effort described here is the first step in this process, namely simulation of the performance of a small DSN. Section II describes this DSN in detail. The setup described will be nearly identical for our physical system. Section III explains the operation of our current algorithm within this DSN. This section also discusses interactions and communications among the heterogeneous nodes of the DSN. Section IV outlines the parameters specific to this simulation and the software used to produce our results. Section V discusses the data produced from these simulations and the implications they hold for detection of small or well-shielded mobile radioactive sources. In Section VI we describe our most recent algorithmic work as well as other issues we plan to investigate in the near future.

\section{HARDWARE}

Our preliminary DSN is composed of arrays of $75 \mathrm{~mm} \mathrm{NaI}$ scintillators, directly connected to PDAsized platforms which provide in situ processing of raw gamma counts. In situ processing is preferred as it eliminates the single point of failure issue and may potentially weed out faulty measurements as well. The detectors are placed several meters apart 
within each array, with an independently operating array on either side of the road to be monitored. In forward and rear positions and interspersed among the radiation detectors are simpler nodes like the well-known mica 2 mote from Crossbow, using accelerometers, magnetometers, etc. for direct vehicular detection and tracking through the DSN operational space. These smaller sensor/processor/radio platforms assist the primary mission by providing data on vehicle presence, localization, and even approximations of speed.

We have chosen PDAs to handle radiation data because we believe that the greater processing capability will be called for to cope with future algorithmic requirements. Additionally a PDA is usually smaller than the radiation detection equipment to which it is attached. Further, PDAs with a generalpurpose operating system (in this case Linux) can utilize familiar and well tested software tools to manipulate and communicate our data.

Figure 1 shows this DSN setup along with the portal monitor alternative. The smaller expendable motes are placed immediately next to the road while the larger radiation detectors are one to two meters farther back. The larger and much taller portal monitors are typically very close to the vehicle, which is not a safety issue at low speeds.

This approach uses small, low-cost, lowefficiency detectors instead of the highly efficient detectors typical in portal monitor systems. Additionally our approach does not require traffic to slow down, whereas portal monitors can usually handle no more than a $10 \mathrm{mph}(4.5 \mathrm{~m} / \mathrm{s})$ vehicle speed. Our DSN approach can perform well for significantly higher speeds. To achieve good performance we will use many detectors (anywhere from tens to thousands, depending on required resolution and desired area of coverage). For coverage of a large area, even portal monitors must be networked much like a DSN in order to coordinate data delivery. Our approach has this ability for scaling built-in, acting more like a single giant detection system than a collection of unrelated sensors.

\section{METHODS}

We assume a typical two-lane road of six to seven meters in width with a posted speed between 25 to 45 miles per hour $(11-20 \mathrm{~m} / \mathrm{s})$. The detectors are set well away from the roadway, such that the two arrays are 10 meters from each other (see Figure 1 ). The speed limit implies that a suspect vehicle will travel at almost exactly the expected velocity in an effort to be inconspicuous. This assumption of constant, known speed eased our analysis methods, but varying and unknown vehicle speeds can be handled by some future refinements.

To compensate for the reduced efficiency and reduced source interaction time of the smaller detectors, we combine the data values (gamma counts) across the detection array coordinated with the motion of the radioactive source. This coherent signal addition uses an integration window that follows the source as it moves past each detector in the array. Algorithm development, described in detail in [5], discovered that when this window length matches detector spacing and expected time lag, increasing the number of detectors also increases the signal-tonoise ratio along a $\sqrt{n}$ curve. When support sensors indicate that there is no traffic, the system collects background radiation measurements and compiles these as a mean and standard deviation. These values are then used to produce a baseline threshold. Vehicles which cause a significantly higher count than this threshold trigger an alert in the system which is propagated out of the network as appropriate.

In our coherent addition method whenever a vehicle approaches, the forward sensors cue the radiation detection apparatus which is otherwise collecting background radiation levels and updating its statistical model of noise. These forward units typically detect either seismic (using an accelerometer) or acoustic (using a microphone) vibrations and broadcast a timestamped report when threshold values are exceeded. This chatter rapidly propagates to the first radiation detector which informs its neighbors of an oncoming target. Note that the motes use a separate radio frequency from the PDAs, but one or more of the PDAs have a mote gateway attached in order to listen in. Using the mote timestamp the first radiation detector begins recording counts for the interaction time that the vehicle is centered on that detector. In other words, the detector begins counting when the vehicle is, for example, 10 meters from its closest approach and ceases when it has receded 10 meters. At this moment, the next detector begins recording readings, and so on down the array. The total gamma count across all channels minus background noise, along 
with local background statistics, is then passed up the line. The end node adds the values, calculates a threshold from the noise statistics, and determines if the total exceeds the threshold. If so, an alert is propagated to an uplink for human intervention.

In practice, we want to limit the number of detectors to about 11 when using this algorithm as described. Source speed will vary and the additive effect on the signal-to-noise ratio then reaches an asymptote at increasingly larger scales. Although work in [5] seems to indicate that small accelerations will not have too large an impact on the signalto-noise ratio. If our support sensors can provide accurate speed estimates, we can space the radiation detectors more widely and dynamically adjust the interaction window as appropriate for the reported speed. With slower sources, the system would act as if it had regular gaps in the array, yet it would be equally effective.

As an alternative, we could cover various constant speeds, accelerating sources and, to a hardwaredependent extent, characterize the spectral signature of these sources using more computationally intensive Bayesian methods, which we discuss in Section VI.

\section{Simulation}

In an effort to thoroughly explore the design space of our DSN and test our software before field tests, we turned to computer simulation. First we defined the environmental circumstances in which our DSN will operate. We assumed a radiation source composed primarily of the Cesium-137 isotope. This isotope was chosen for its industrial availability, its extremely high radioactivity, and its typical powdered form as cesium chloride $(\mathrm{CsCl})$, all of which promote its use in an RDD.

Our simulation also assumed a speed limit of 45 $\mathrm{mph}(20 \mathrm{~m} / \mathrm{s})$. Given this expected speed, we placed the 11 detectors at intervals of 20 meters within each array and we set the detection integration time (i.e., how frequently a detector reports its gamma count) at one second.

We also assumed a very small mass of cesium which is unshielded. The detectability of this source is equivalent to a larger measure of the isotope in a lead container of significant thickness. By choosing a very small amount of radioactive material, we tested the limits of our detection scheme. In order to bypass shielding effects, we describe the sources in terms of their activity in curies $(\mathrm{Ci})$ : specifically $.01 \mathrm{Ci}$ and $.1 \mathrm{Ci}$. Finally, we also simulated the larger and technically more efficient portal monitor approach as a comparator.

Our simulation software consists of the Distributed Sensors Simulator (DSS), an in-house DSN simulation framework, plus a fast radiation propagation and detection spectral synthesizer from $\mathrm{Pa}$ cific Northwest National Laboratory (PNNL) named Synth. DSS was created to support our DSN software development process. It simulates wireless communications, source propagation, and DSN topology for multiple, arbitrary applications [2]. DSS accepts user specifications of node characteristics, source characteristics, functions representing source propagation, and simulated node failures, as well as the application software that will run in the virtual nodes. DSS is awaiting approval for open source release from the U.S. Department of Energy.

Synth models gamma propagation using hardcoded algorithms as opposed to Monte Carlo methods, producing even complex spectra fairly rapidly [3]. Synth also provides simulated background readings plus statistical fluctuations, while allowing for a variety of detector types. Synth is commercially available from PNNL.

Using DSS, coupled with Synth to provide radiation propagation, we simulated the performance of the algorithm described in Section III on the hardware of Section II with the passing sources mentioned above. We also simulated the same sources as detected by a representative portal monitor package with an integration time of 11 seconds. This typical portal monitor consists of the more efficient five inch by two inch $(13 \times 5 \mathrm{~cm}) \mathrm{NaI}$ crystal. Generally, portal monitors employ plastic scintillators which may be more efficient than this $\mathrm{NaI}$ detector due to their large surface area. However several commercially available portal monitors do utilize $\mathrm{NaI}$ crystals similar to our example, which leads us to conclude that it is sufficiently typical of the detection capability of portal monitors.

\section{RESUlts}

We expected our simulations to merely show the suitability of our DSN approach, since DSNs are more appropriate for certain detection deployments. Instead we have found that the DSN option promises 
improved performance over a single detector to the point of being comparable to some portal monitors, while avoiding the vehicle speed caveat.

We compared performance across three $\mathrm{NaI}$ detector sizes to evaluate contenders for the radiation detection component of our DSN. The $75 \mathrm{~mm} \mathrm{NaI}$ radiation detector is a very common detector in general. The $50 \mathrm{~mm}$ and $25 \mathrm{~mm} \mathrm{NaI}$ detectors might be preferable for their smaller size and cost savings. We wished to establish and quantify the multiplicative effect of our method as well. These three goals come through very clearly in the resultant data.

Radiation detection nodes are cued when the forward support sensors detect a vehicle at closest approach, and we consider this to be $t_{0}$. Per Figure 1 this occurs at two and a half seconds before the vehicle passes the first radiation detector at a velocity of $20 \mathrm{~m} / \mathrm{s}$. At two seconds into our sequence, $\mathrm{t}_{2}$, radiation detector number one begins count collection which ceases at $t_{3}$. Detector two starts at $t_{3}$ and ends at $t_{4}$, and so on. Each radiation detector senses an energy spectrum similar to the red line in Figure 2, which is an average, except with the inclusion of background. The detector hardware sums the values of these channels which is passed to the PDA. The PDA subtracts out the collected background to get a value as seen by the bar representing the $75 \mathrm{~mm}$ single detector in Figure 3 , which again is an average. This value and the single detector background standard deviation value from Figure 3 are passed to the next node, which will in turn append its data to the message. At the final node, the counts are summed to get the $75 \mathrm{~mm}$ DSN array value in Figure 3 and the background deviations are summed, which is also represented in the figure. If the total count exceeds, in this case, two standard deviations of the background, an alert is signalled.

Figure 2 shows the nearly equivalent spectra produced by the portal monitor, DSN radiation detection by coherent addition, and detection of an order of magnitude larger source by a single DSN class detector. These spectra are separated from the ever-present background count. This result is important for two reasons. First, it demonstrates that our DSN approach is equivalent to, and possibly better than the portal monitor approach because of the higher counts at each channel. Second, it shows the efficacy of coherent addition, yielding a signal nearly identical to that produced in detecting a source 10 times larger. Although the spectra are not used by this algorithm, they do provide insight as to how coherent addition is better and what it takes to get this performance gain over a given system. Ignoring statistical fluctuations and the caveat of constant speed, our method essentially multiplies the spectra produced by a single detector by the number of detectors in the array.

In Figure 3, we compare the capabilities of three $\mathrm{NaI}$ detector sizes: $75 \mathrm{~mm}, 50 \mathrm{~mm}$, and $25 \mathrm{~mm}$. Over the course of the coherent addition algorithm, we collect a total count across all channels exclusive of background. The DSN approach shows marked improvement over the portal monitor in Figure 3. This figure indicates that the $75 \mathrm{~mm}$ DSN detector is distinctly better than the portal monitor over the same total system integration time. The significantly less efficient and less expensive $50 \mathrm{~mm} \mathrm{NaI}$ detector, also in a coherent additive array of 11 sensors, collects a total count that appears to still be sufficient for our purposes. This is so because it is considerably more than one, or even several, standard deviations of the sampled background.

For a small .01 curie source, the $25 \mathrm{~mm}$ detector will be inadequate even with coherent addition. This demonstrates a limitation of our approach: the system cannot improve resolution if the signal collected by component detectors is not significant. An individual $75 \mathrm{~mm}$ detector is small enough to be convenient, yet an array of 11 outperforms a single portal monitor. Since we are willing to use many detectors, we can surpass a given performance ceiling and achieve that performance for much faster sources than is typical.

The fact that our approach compares very well with the bulky alternative in simulation is reassuring and leads us to conclude the general worthiness of a DSN approach. The next step is to prove this in hardware which, along with algorithmic improvements, is our goal for the coming months.

\section{FURTHER WORK}

As mentioned above, our next step is demonstration and validation with real detectors and a variety of real sources. Enhancement of our signal processing techniques is a continuing parallel effort. We now are developing a new technique using Bayesian methods that allows for source acceleration with even better performance than our coherent addition approach. 
Generally, Bayesian methodology determines the probability of the resultant data from a collection of classes of possible origins as detailed in [1]. We have two such classes. Our first class consists of all 20-second long trajectories parallel to the two longest sides of a rectangle representing the road: the events we wish to detect. The second class consists of 20-second long trajectories which are not incident with the rectangle. In the simulations, a source has equal probability of belonging in either class. Velocities ranging between $10 \mathrm{~m} / \mathrm{s}$ and 30 $\mathrm{m} / \mathrm{s}$ are considered equally probable. Any source amplitude in curies below a maximum are also taken to be equally probable. The counts recorded at a sensor are assumed to be Poisson distributed about a mean which is dependent on detector coordinates, source amplitude, velocity and background radiation. The section of road we are monitoring is again represented as a rectangle with a 10-meter width and this time a length of 600 meters, as seen in Figure 4.

In a Fortran simulation, we examined three detection approaches, each containing 10 sensors in different configurations. The first configuration (random) was generated by pseudo-random uniform sampling within the rectangle. The second configuration (portal monitor) has the 10 sensors equally spaced along the intersection of the rectangle with the $y$ axis. The third configuration (shoulder) places the 10 sensors along the long edges of the rectangle, equally spaced and staggered. We compiled the true positive detection rates (sources we should detect and do detect) and true negative rates (nonsource events we should not and do not detect) for trajectories derived from the two classes as seen in Table I.

\begin{tabular}{l|cc}
\hline Placement & True positives & True negatives \\
\hline portal monitor & .57 & .89 \\
one shoulder & .74 & .92 \\
both shoulders & .76 & .93 \\
random & .91 & .96
\end{tabular}

TABLE I

PERFORMANCE OF VARIOUS SENSOR PLACEMENT APPROACHES USING A SIMPLE BAYESIAN TECHNIQUE.

This data reinforces our belief that DSN topologies perform better than portal monitoring, and it indicates something new. The best performance is gained by placing detectors randomly on the road. For reasons of safety, this means that the detectors must be embedded flush with the asphalt, or otherwise removed from the path of traffic. This is a possibility that bears further investigation. If we are to use this method however we will need to find ways to reduce its computational intensity which is considerable.

In order to expedite further development of our approach, we will next seek to determine how well our simulation software does its job. By comparing a variety of data derived from physical experimentation with results from the simulator, we hope to establish a measure confidence in the accuracy of the simulator and a means of calibrating it with real data.

\section{CONCLUSION}

Expanding on the work in [6] and [4], which suggest that bigger is not necessarily better in radiation detectors, we are seeking to provide flexible, discreet detection solutions that enhance not just national security, but global nonproliferation. We wanted to demonstrate niche applications for DSN radiological detection, and found that there is great potential for DSNs to play a much more significant role. Radiological DSNs can complement the portal monitor approach by enabling rapid deployment and much greater transparency to the public while achieving equal or greater performance. Distributed sensor networks show promise not just for radiation detection and rapid response, but for in situ and real-time detection of a multitude of dangerous phenomena. In the coming years, as sensor hardware improves, we will expand our efforts to reduce other threats such as chemical or biological weapons.

\section{ACKNOWLEDGMENT}

This work was supported by the U.S. Department of Energy/NNSA and Los Alamos National Laboratory funds under University of California Contract W-7405-ENG-36.

Approved for public release under LA-UR-04xxxx.

\section{REFERENCES}

[1] J. O. Berger and D. A. Berry, "Statistical Analysis and the Illusion of Objectivity," American Scientist, 76, pp.159-165, 1988. 
[2] S. M. Brennan, Distributed Sensor Network Software Development: Testing through Simulation, Master's thesis, University of New Mexico, 2003.

[3] W. K. Hensley, Synth: A Gamma-Ray Spectrum Synthesizer, http://www.pnl.gov/fi ber/synth.html, updated December 20, 1996.

[4] M. A. Nelson, B. D. Rooney, Modeling and Bench-Top Results of the Arrayed Vehicle Monitor, Los Alamos National Laboratory report LA-UR-03-8055, 2003.

[5] R. J. Nemzek, J. S. Dreicer, D. C. Torney, and T. T. Warnock , Distributed Sensor Networks for Detection of Mobile Radioactive Sources, Los Alamos National Laboratory report LA-UR-038557, 2003

[6] K. P. Ziock and W. H. Goldstein, "The lost source, varying backgrounds and why bigger may not be better," in Unattended Radiation Sensor Systems for Remote Applications. J. I. Trombka, Ed., New York: American Institute of Physics, 2002.

Sean M. Brennan is a graduate research assistant at Los Alamos National Laboratory. His research interests include scalability and fault tolerance of distributed computing and sensory systems, as well as practical deployment issues. He received an MS degree in computer science from the University of New Mexico and is currently working toward a $\mathrm{PhD}$. He is an IEEE student member. Contact him at brennan@lanl.gov.

Arthur B. Maccabe is a professor at the Computer Science Department of the University of New Mexico. He received a $\mathrm{PhD}$ in Information and Computer Sciences from the Georgia Institute of Technology. Contact him at maccabe@cs.unm.edu.

Angela M. Mielke is the project leader for the NNSA-funded Distributed Sensor Networks with Collective Computation Project at Los Alamos National Laboratory. Her primary research experience is in the design and algorithm development of statistical pattern recognition techniques for data analysis and discovery. She received an Electrical Engineering degree from Villanova University. Contact her at amielke@lanl.gov.

David C. Torney is a technical staff member at Los Alamos National Laboratory. He is an applied mathematician with a $\mathrm{PhD}$ in Biophysics and Mathematics from Stanford University. Contact him at dct@lanl.gov. 


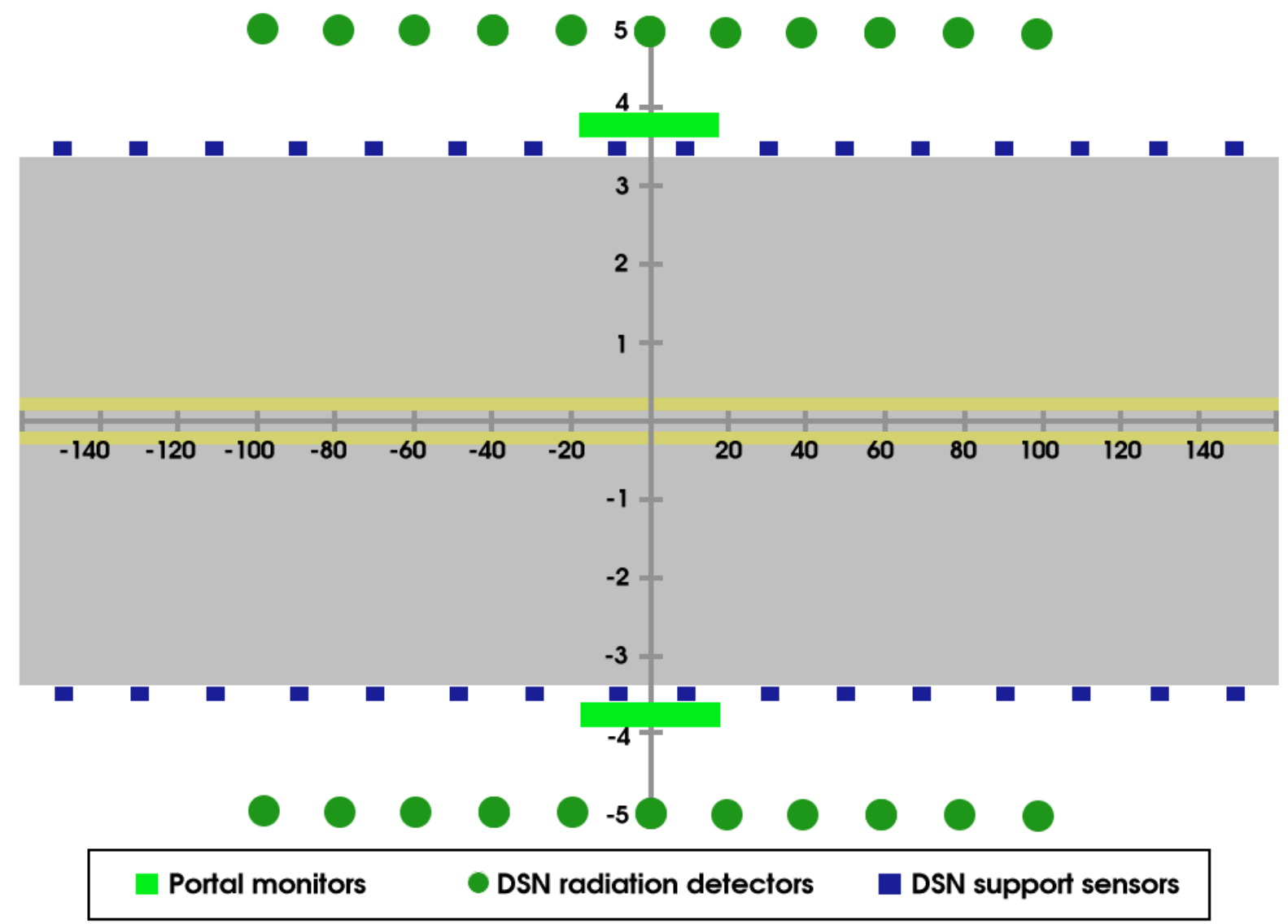

Fig. 1. Example radiation detection DSN topology. DSN radiation detectors are coupled with PDAs which signal an alert via an uplink. Portal monitors have a direct uplink. All measurements are in meters. 


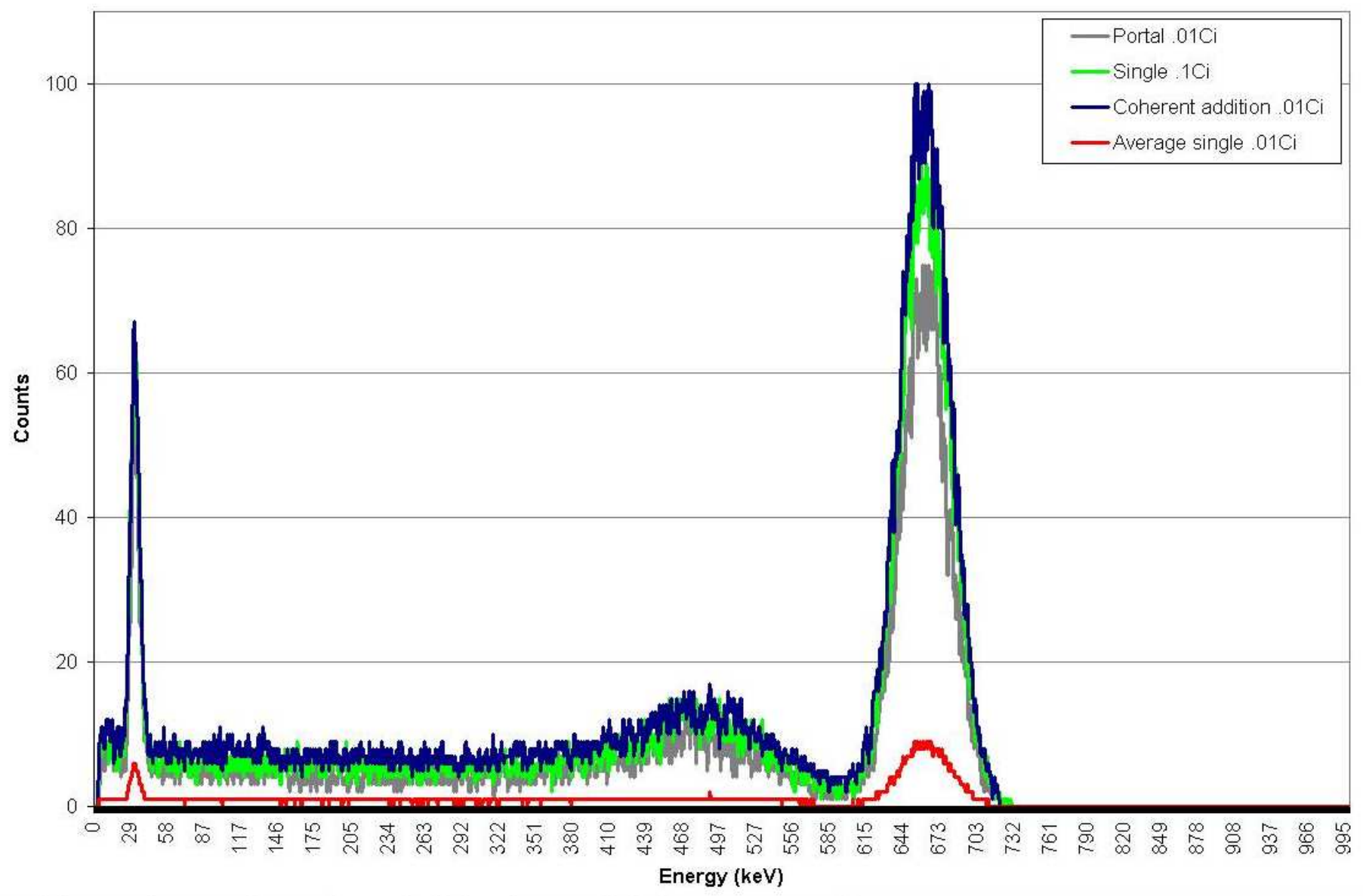

Fig. 2. Detected spectra for coherent addition of eleven $75 \mathrm{~mm} \mathrm{NaI} \mathrm{DSN} \mathrm{detectors} \mathrm{with} \mathrm{a} 0.01 \mathrm{Ci}$ source, a typical portal monitor detection of the same source, and detection of a $0.1 \mathrm{Ci}$ source using only one DSN detector, as well as an average single DSN detector sensing a 0.01 Ci source. 


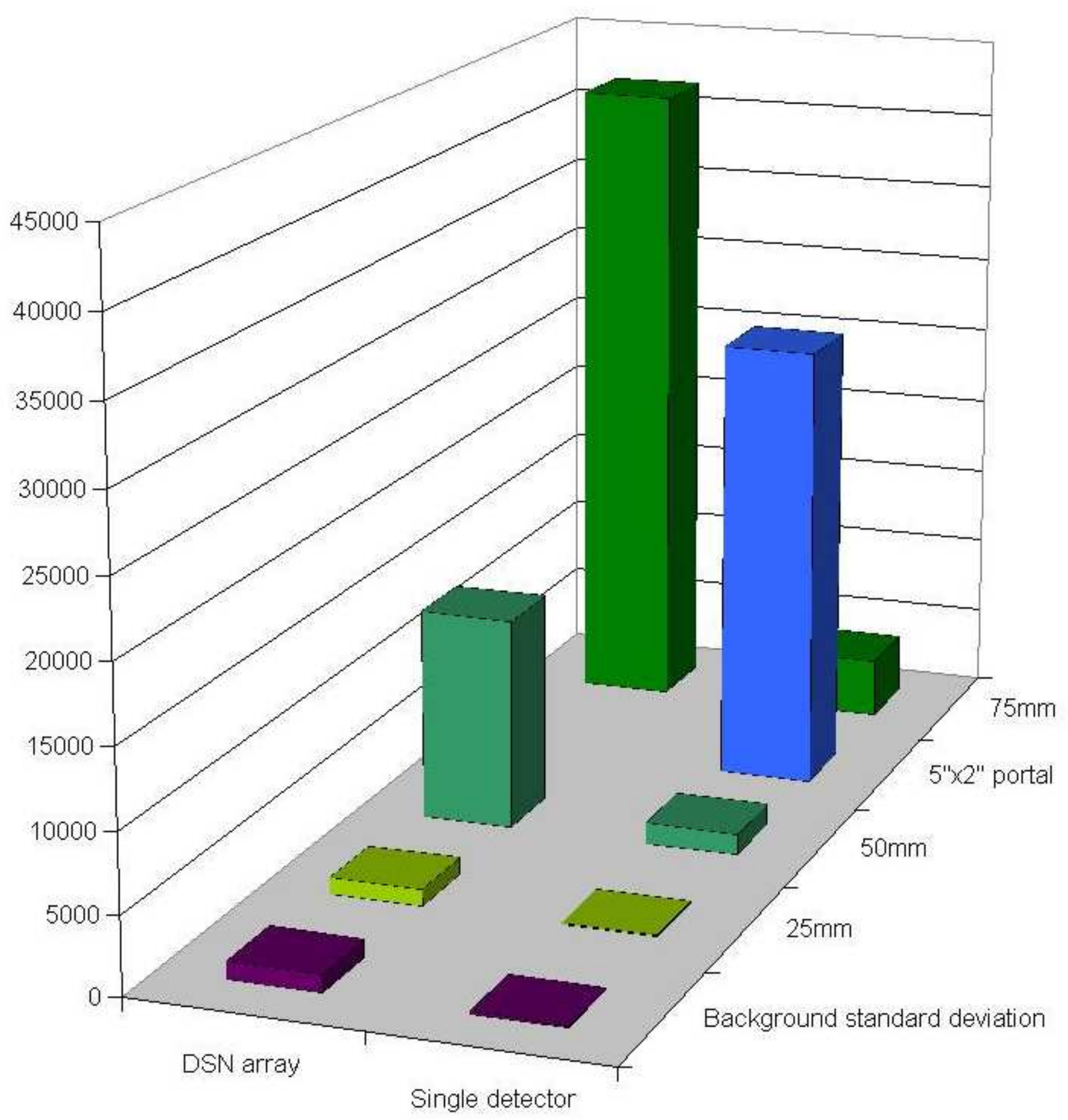

Fig. 3. Performance of various detectors. The integration time for one detector is 1 second, yielding a total of 11 seconds for an array. The portal monitor integration time is 11 seconds. Background standard deviation for the portal monitor is equivalent to that of the DSN array. 


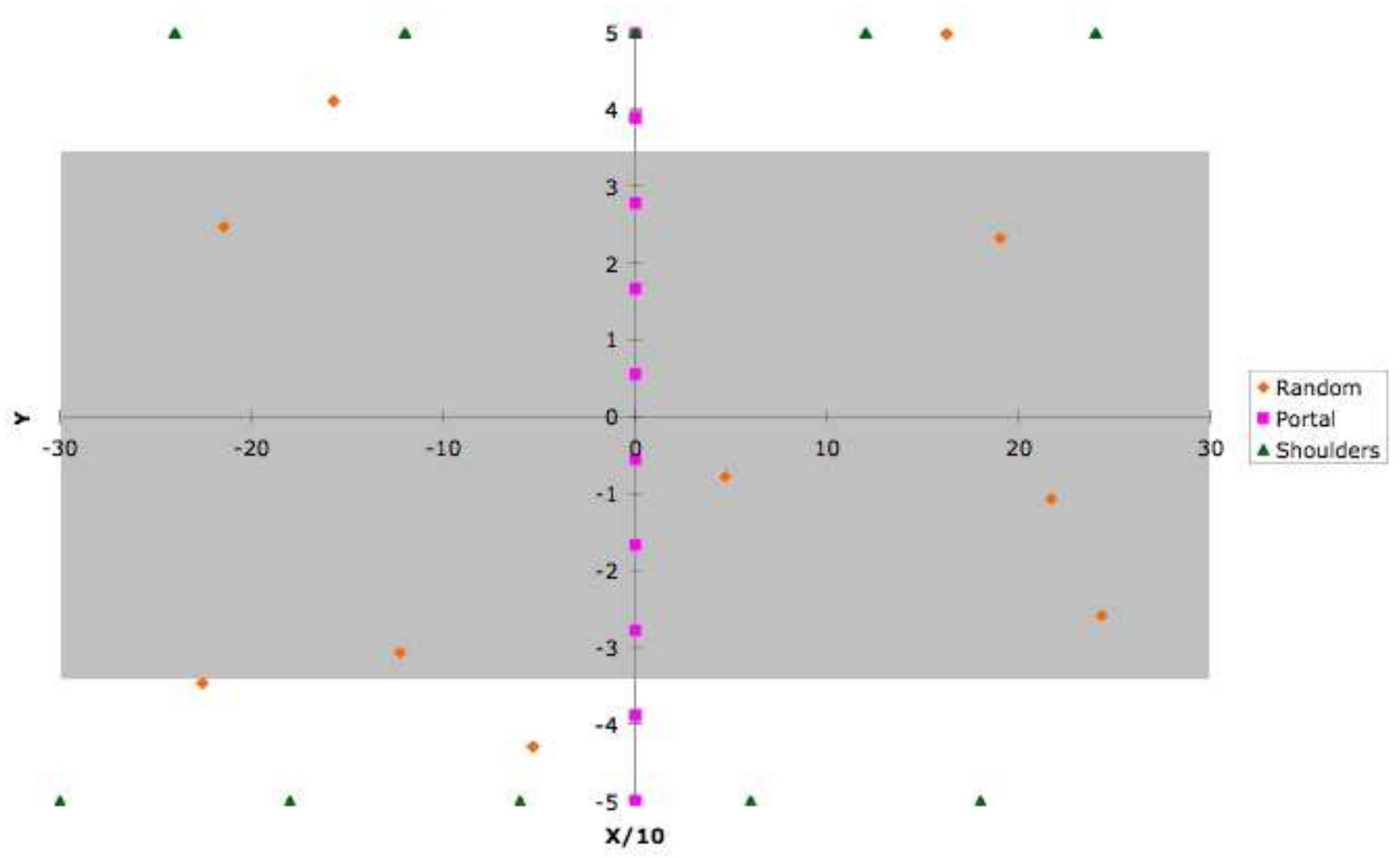

Fig. 4. Detector placement in and around a two-lane road for using our Bayesian method. 